\title{
Inflammatory breast cancer: no longer an absolute contraindication for breast conservation surgery following good response to neoadjuvant therapy
}

\author{
Monika Brzezinska, J. Michael Dixon \\ Edinburgh Breast Unit, NHS Lothian, Western General Hospital, Edinburgh, UK \\ Contributions: (I) Conception and design: All authors; (II) Administrative support: None; (III) Provision of study materials or patients: None; (IV) \\ Collection and assembly of data: None; (V) Data analysis and interpretation: None; (VI) Manuscript writing: All authors; (VII) Final approval of \\ manuscript: All authors. \\ Correspondence to: Professor J. Michael Dixon. Edinburgh Breast Unit, NHS Lothian, Western General Hospital, Crewe Road South, Edinburgh \\ EH4 2XU, UK. Email: mike.dixon@ed.ac.uk.
}

\begin{abstract}
Inflammatory breast cancer (IBC) is a rare form of breast cancer characterised by an erythematous swollen breast with extensive oedema and has in the past been associated with a very poor prognosis. After diagnosis by core biopsy of the cancer and any involved nodes patients in the Edinburgh Breast Unit (EBU) are primarily managed with neoadjuvant systemic therapy—chemotherapy or endocrine therapy. If the cancer is localised to one or a few well defined lesions then each of these lesions together with the lowest involved node are clipped. Response during neoadjuvant treatment is monitored clinically and by ultrasound plus mammography +/- magnetic resonance imaging (MRI). Following completion of neoadjuvant therapy, imaging is reviewed at a multidisciplinary meeting and patients with a localised single or multiple areas of cancer where all signs of erythema and oedema have settled are considered as to their suitability for breast conserving surgery and whole breast radiotherapy [breast conserving treatment (BCT)]. Here we discuss the results and outcomes of a selected group of patients with IBC who after obtaining a very good response to neoadjuvant chemotherapy or endocrine therapy were treated by BCT and we compare these with other recent publications on this topic. Our data show that patients treated by BCT did not have worse outcomes than patients treated with mastectomy. Importantly other series published recently support our conclusions. Another important observation is that response in estrogen receptor (ER) rich IBC is seen with neoadjuvant endocrine treatment and so not everyone with IBC needs to have neoadjuvant chemotherapy. In conclusion, patients with one or more well defined and localised breast masses and IBC may be suitable for BCT after a major response to neoadjuvant therapy and for these patients BCT should now be considered a viable option.
\end{abstract}

Keywords: Inflammatory breast cancer (IBC); breast conserving treatment (BCT); neoadjuvant chemotherapy (NAC); neoadjuvant hormone therapy

Submitted Feb 14, 2018. Accepted for publication Aug 17, 2018.

doi: 10.21037 /gs.2018.08.04

View this article at: http://dx.doi.org/10.21037/gs.2018.08.04

\section{Introduction}

Inflammatory breast cancer (IBC) is one of the rarest phenotypes and comprises between $1-5 \%$ of all newly diagnosed breast cancers $(1,2)$. It tends to affect younger women with a median age at diagnosis of 52 years as reported in a UK retrospective multi-institutional study; this contrasts with an average age of 57 years in a US study (3). Various classification systems have been used to define inflammatory breast cancer following the first detailed description by Haagensen in 1956 (4). The criteria 
for a diagnosis of IBC outlined by Haagensen form the basis of the definition of IBC by the American Joint Committee on Cancer (AJCC). IBC is considered a "clinico-pathological entity characterised by diffuse erythema and oedema of the breast, often without an underlying palpable mass". The associated oedema results in an enlarged breast and a characteristic clinical feature is widespread peau d'orange $(5,6)$. The International Guidelines and AJCC guidelines 7th edition and 8th edition state that both erythema and oedema should be present and occupy at least one-third of the skin of the breast. IBC is classified as T4d in the TNM classification and the diagnosis of IBC is based primarily on its clinical features. According to the 2012 WHO classification a diagnosis of IBC requires typical clinical and/or specific histological features (7). This classification also requires that the characteristic changes of the skin be of rapid onset (less than 6 months) (8). Radiologically, other than marked skin thickening, the most common feature is an absence of a well-defined breast mass on imaging and any visualised disease is frequently involving more than one area of disease in the breast. A minority of IBC patients have a localised mass or more than one area of cancer close to each other. Advances in imaging techniques including high frequency ultrasonography, magnetic resonance imaging (MRI) and positron emission tomography/computed tomography (PET/CT) have improved the consistency of diagnosis and staging of $\operatorname{IBC}(9,10)$. Such imaging allows mapping of invasive disease and MRI is considered the most accurate way of assessing IBC at diagnosis and also in monitoring response to treatment, which helps to guide subsequent surgery $(11,12)$.

Inflammatory breast cancer does not have a specific histological appearance and in particular there are no classical features on microscopy of an inflammatory process. One of the pathological hallmarks of IBC is the presence of tumour emboli in lympho-vascular spaces; these are composed of clumps of tumour cells within vascular or more commonly lymphatic spaces in the dermis, but the presence of these is neither necessary nor sufficient for a diagnosis of IBC (13). It has been suggested that punch biopsy of the skin should be a standard investigation in suspected IBC and that tumour in dermal lympho-vascular spaces should be a requirement for diagnosing IBC but dermal lymphatic invasion although helpful in establishing the diagnosis is not seen in approximately $30 \%$ of cases (14). In most cases the cancer is classified histologically as being of no special type and usually IBC is usually grade 3 and consists of cancer cells that are pleomorphic with high nuclear grade (15). Invasive lobular cancers represent only between $4.5-5 \%$ of IBCs (16). IBC has higher proportion of HER2 receptor over-expression than seen in noninflammatory cancers or other locally advanced cancers (17). Given the high rate of HER2 positivity in IBC anti HER2 directed treatments have been shown to be very effective in HER2 positive IBC. In the NOAH trial of neoadjuvant trastuzumab combined with chemotherapy, HER2 positive IBC cancers had a pathological complete response (PCR) rate of $54.8 \%$ (18). A similar PCR rate of $45.8 \%$ in IBC was reported in the NeoSphere trial with the addition of pertuzumab to trastuzumab and chemotherapy. This was much higher that the PCR rate of $29 \%$ of patients with IBC in this study who received only trastuzumab together with docetaxel neoadjuvant chemotherapy (NAC) (19). Patients only received 12 weeks of treatment in the NeoSphere trial so it may be that the longer duration of chemotherapy and trastuzumab in the NOAH study explains the difference in PCR rates between these two studies.

Epidermal growth factor receptor (EGFR) expression has been observed in $30 \%$ of IBC and has been associated with worse prognosis and an increased risk of IBC recurrence (20). IBC is also known to have high rates of endothelial cell proliferation and vascular density in comparison to other types of breast cancer which suggests there could be potential role for anti-angiogenic therapies. Cristofanili et al. reported the results of a multi-centre phase 2 study evaluating lapatinib and pazopanib in patients with relapsed HER2 positive IBC (21). The combination of these agents, although associated with greater toxicity produced a higher overall response rate but no increase in progression free survival compared to lapatinib alone. This raises the possibility of incorporating new anti-angiogenic therapies in to future chemotherapy regimens in IBC.

About $85 \%$ of patients with inflammatory breast cancer at the time of diagnosis have metastases to regional lymph nodes, and almost $30 \%$ have distant metastases (6). The average overall 5 -year survival for patients with IBC is less than $55 \%$ (22).

IBC has been historically treated by mastectomy followed by chest wall radiotherapy often with skin bolus yet despite maximal surgery and radiation many women still have a poor prognosis. The addition of NAC has improved overall survival (OS) rates. Currently, the ESMO and international panel recommendation is to perform total mastectomy and axillary dissection regardless of any response to neoadjuvant treatment $(8,23)$. Recently, UK guidelines (The UK IBC working group, 2016) (24) suggest that in IBC patients 
who respond well to primary systemic chemotherapy breast conserving treatment (BCT) consisting of breast conserving surgery (BCS) and whole breast radiotherapy can be performed. More effective NAC has resulted in improved tumour response rates and with the use of MRI to accurately evaluate response of treatment, it is not surprising that $\mathrm{BCS}$ is now considered a viable option for selected patients with IBC. Bonev et al. reported seven IBC patients who underwent BCS and compared them with 24 patients with IBC who underwent mastectomy. They found no significant difference between the two groups in the OS rate after 60 months follow up (25). In a large SEER study of 3,374 patients with inflammatory breast cancer there was no significant difference in OS and breast cancer specific survival in patients treated with BCT (150 patients) compared to the mastectomy group (26).

\section{Results and discussion}

In the EBU patients with IBC are managed initially by neoadjuvant systemic therapy. It is our routine to clip tumour masses if single or localised to one area of the breast and also to clip the lowest involved node after proving nodal involvement by core biopsy or fine needle aspiration (FNA). We feel it is important not to prejudge which patients might be suitable for BCS at diagnosis as response depends more on cancer phenotype than cancer extent. Depending on the patient's age, general health and tumour characteristics neoadjuvant treatment can be either NAC or neoadjuvant endocrine therapy-used in postmenopausal women often with other co morbidities whose cancers are ER rich (Allred scores 7 or 8). For many years in Edinburgh there has been the view that in a carefully selected subgroup of patients with localised IBC who get an excellent response to neoadjuvant treatment characterised by disappearance of all erythema and oedema that BCT can be offered (27). We screened our prospectively collected database and identified 35 patients between 1999 and 2013 who were diagnosed with IBC and who were treated with BCT after neoadjuvant therapy. This group of patients had localised tumour either as single mass or 2 or more masses close to each other and were treated initially with NAC or neoadjuvant endocrine therapy and subsequently had BCS and adjuvant whole breast radiotherapy. Patients were monitored during their neoadjuvant therapy with regular ultrasound plus mammography +/- MRI and all women were discussed at a multidisciplinary meeting prior to surgery. If the clinical symptoms (erythema, peau d'orange) of the IBC had resolved and response was evident on imaging and there was a localised mass or masses that the surgeon thought could be excised and that excision would leave a reasonable cosmetic result then BCT was offered to the patient. The BCS performed consisted of a wide local excision of any residual mass or needle localisation wide excision of any clips and was usually combined with axillary surgery. Radial margins on the wide excision had to be clear of tumour by $1 \mathrm{~mm}$ or more or re excision was advised.

Axillary lymph node dissection (ALND) was performed in 20 patients. Sentinel lymph node biopsy (SLNB) including removal of any clipped node was performed in 14 patients and one patient was treated with axillary radiation without any axillary surgery. Four patients in the SLNB group had completion ALND. Our SLNB identification rate was $100 \%$. We routinely used a dual technique with blue dye and radioisotope and have always taken 3 or more nodes. Adjuvant whole breast irradiation followed BCT in all the patients.

We analyzed our data and we calculated the locoregional recurrence (LRR)-free survival rates and these gave an $87.5 \%$ 5-year LRR-free survival and actuarial 5-year survival rate of $70.3 \%$ with a median follow up of the cohort being 80 months. It is worth noting that the OS in the whole IBC patients group treated in the EBU was $61.9 \%$. This is better than that usually reported and shows how with modern neoadjuvant therapies and carefully performed surgery and radiation patients with IBC can now expect long term survival.

Over $60 \%$ of our patients' cancers in the Edinburgh series treated by BCT were strongly ER positive and as discussed above in Edinburgh we have pioneered the use of neoadjuvant endocrine therapy as well as NAC. Reponses to neoadjuvant endocrine therapy were as impressive as those seen with NAC suggesting that this is an option for older women with ER rich cancers. A meta-analysis of NAC and neoadjuvant endocrine therapy in breast cancer reported similar rates of response but higher rates of BCS with endocrine therapy (28). This is because of the histological pattern of response seen with neoadjuvant endocrine therapy where central scarring with tumour implosion is seen in almost two thirds of cancers treated by neoadjuvant endocrine therapy but this pattern is rarely seen with NAC (29). This results in the cancer shrinking concentrically rather than breaking up, a pattern more common with chemotherapy. This concentric shrinkage 
makes the cancer easier to remove and get clear margins when performing BCS after neoadjuvant endocrine therapy. At a median follow-up of over 8 years' excellent rates of local and systemic control were seen in the patients having BCT and fully justified our use of both neoadjuvant endocrine therapy and BCT. All but one of the patients with local recurrence had metastatic disease at the same time or within a few months of the local recurrence being diagnosed. This indicates that the biology of the disease determines recurrence much more that the surgery performed. The other patient with a recurrence was found to be a $B R C A 1$ gene carrier and the recurrence was a second cancer and after further surgery remains well.

Our data and those from other studies (26) indicate that it is no longer mandatory to state that inflammatory cancer is an absolute contraindication to BCT. Likewise, after neoadjuvant endocrine or chemotherapy SLNB is not in our view absolutely contra indicated. The art of medicine is selecting the appropriate patients for the most appropriate treatments. We are not surprised that others have struggled to find sentinel nodes after NAC in IBC. We would only consider performing SLNB in selected patients with either no evidence of node involvement at diagnosis or an excellent response in involved nodes. It needs to be stressed that the majority of patients with IBC are not suitable for BCT or for SLNB following neoadjuvant systemic therapy, but for those who do respond well then it is our view that BCS and SLNB followed by whole breast radiotherapy should be considered as it does not appear to result in a worse outcome. Breast cancer treatment continues to evolve. It is important that as we do for systemic therapy that we individualise local treatment. The concern that doing less might lead to a greater local recurrence rate and a worse survival has been proven to be incorrect. A better understanding of individual cancer phenotypes has resulted in better directed systemic therapy with higher rates of PCR to neoadjuvant treatment. This raises the possibility that for those women with such a response we can de-escalate local treatments. This report represents a step in that direction.

So, what for the future? A further collection of multicenter data is required from women with IBC who have one or more localised breast masses who achieve a good response to systemic therapy with disappearance of erythema and oedema who are treated by BCT to confirm our finding that this approach is safe. Then in the knowledge every unit will be able to offer selected patients with IBC the option of breast preservation having proven that it is a safe option.

\section{Acknowledgements}

None.

\section{Footnote}

Conflicts of Interest: The authors have no conflicts of interest to declare.

\section{References}

1. Anderson WF, Schairer C, Chen BE, et al Epidemiology of inflammatory breast cancer (IBC). Breast Dis 20052006;22:9-23.

2. Goldner B, Behrendt CE, Schoellhammer HF, et al. Incidence of inflammatory breast cancer in women, 19922009, United States. Ann Surg Oncol 2014;21:1267-70.

3. Copson E, Shaaban A, McKenzie H, et al. Inflammatory Breast Cancer: the UK experience. 2015 NCRI Cancer Conference, Liverpool, UK.

4. Haagensen CD. Diseases of the female breast. Trans N Engl Obstet Gynecol Soc 1956;10:141-56.

5. Greene FL, Page DL, Fleming ID, et al. AJCC cancer staging manual. 6th edition. New York, NY: Springer, 2002.

6. Walshe JM, Swain SM. Clinical aspects of inflammatory breast cancer. Breast Dis 2005-2006;22:35-44.

7. Lakhani SR, Ellis IO, Schnitt SJ, et al. WHO Classification of Tumours of the Breast. Lyon: WHO IARC, 2012.

8. Dawood S, Merajver SD, Viens P, et al. International expert panel on inflammatory breast cancer: consensus statement for standardized diagnosis and treatment. Ann Oncol 2011;22:515-23.

9. Yang WT, Le-Petross HT, Macapinlac H, et al. Inflammatory breast cancer: PET/CT, MRI, mammography, and sonography findings. Breast Cancer Res Treat 2008;109:417-26.

10. Groheux D, Giacchetti S, Delord M, et al. 18F-FDG $\mathrm{PET} / \mathrm{CT}$ in staging patients with locally advanced or inflammatory breast cancer: comparison to conventional staging. J Nucl Med 2013;54:5-11.

11. Dall BJ, Vinnicombe S, Gilbert FJ. Reporting and management of breast lesions detected using MRI. Clin Radiol 2011;66:1120-8.

12. Uematsu T. MRI findings of inflammatory breast cancer, locally advanced breast cancer, and acute mastitis: T2-weighted images can increase the specificity of inflammatory breast cancer. Breast Cancer 2012;19:289-94. 
13. Fouad TM, Kogawa T, Reuben JM, et al. The role of inflammation in inflammatory breast cancer. Adv Exp Med Biol 2014;816:53-73.

14. Charpin C, Bonnier P, Khouzami A, et al. Inflammatory breast carcinoma: an immunohistochemical study using monoclonal anti-pHER-2/neu, pS2, cathepsin, ER and PR. Anticancer Res 1992;12:591-7.

15. Lê MG, Arriagada R, Contesso G, et al. Dermal lymphatic emboli in inflammatory and noninflammatory breast cancer: a French-Tunisian joint study in 337 patients. Clin Breast Cancer 2005;6:439-45

16. Raghav K, French JT, Ueno NT, et al. Inflammatory Breast Cancer: A Distinct Clinicopathological Entity Transcending Histological Distinction. PLoS One 2016;11:e0145534.

17. Zell JA, Tsang WY, Taylor TH, et al. Prognostic impact of human epidermal growth factor-like receptor 2 and hormone receptor status in inflammatory breast cancer (IBC): analysis of 2,014 IBC patient cases from the California Cancer Registry. Breast Cancer Res 2009;11:R9.

18. Gianni L, Eiermann W, Semiglazov V, et al. Neoadjuvant chemotherapy with trastuzumab followed by adjuvant trastuzumab versus neoadjuvant chemotherapy alone, in patients with HER2-positive locally advanced breast cancer (the NOAH trial): a randomised controlled superiority trial with a parallel HER2-negative cohort. Lancet 2010;375:377-84.

19. Gianni L, Pienkowski T, Im YH, et al. 5-year analysis of neoadjuvant pertuzumab and trastuzumab in patients with locally advanced, inflammatory, or early-stage HER2-positive breast cancer (NeoSphere): a multicentre, open-label, phase 2 randomised trial. Lancet Oncol 2016;17:791-800

20. Cabioglu N, Gong Y, Islam R, et al. Expression of growth factor and chemokine receptors: new insights in the biology of inflammatory breast cancer. Ann Oncol 2007;18:1021-9.

21. Cristofanilli M, Johnston SR, Manikhas A, et al. A

Cite this article as: Brzezinska M, Dixon JM. Inflammatory breast cancer: no longer an absolute contraindication for breast conservation surgery following good response to neoadjuvant therapy. Gland Surg 2018;7(6):520-524. doi: 10.21037/ gs.2018.08.04 randomized phase II study of lapanitib + pazopanib versus lapanitib in patients with HER2 + inflammatory breast cancer. Breast Cancer Res Treat 2013;137:471-82.

22. Rueth NM, Lin HY, Bedrosian I, et al. Underuse of trimodality treatment affects survival for patients with inflammatory breast cancer: an analysis of treatment and survival trends from the National Cancer Database. J Clin Oncol 2014;32:2018-24.

23. Cardoso F, Senkus E, Costa A, et al. 4th ESO-ESMO International Consensus Guidelines for Advanced Breast Cancer (ABC 4). Ann Oncol 2018;29:1634-57.

24. Rea D, Francis A, Hanby AM, et al. Inflammatory breast cancer: time to standardise diagnosis assessment and management, and for the joining of forces to facilitate effective research. Br J Cancer 2015;112:1613-5.

25. Bonev V, Evangelista M, Chen JH, et al. Long-term follow-up of breast-conserving therapy in patients with inflammatory breast cancer treated with neoadjuvant chemotherapy. Am Surg 2014;80:940-3.

26. Chen H, Wu K, Wang M, et al. A standard mastectomy should not be the only recommended breast surgical treatment for non-metastatic inflammatory breast cancer: A large population-based study in the Surveillance, Epidemiology, and End Results database 18. Breast 2017;35:48-54.

27. Brzezinska M, Williams LJ, Thomas J, et al. Outcomes of patients with inflammatory breast cancer treated by breast-conserving surgery. Breast Cancer Res Treat 2016;160:387-91.

28. Spring LM, Gupta A, Reynolds KL, et al. Neoadjuvant Endocrine Therapy for Estrogen Receptor-Positive Breast Cancer A Systematic Review and Meta-analysis. JAMA Oncol 2016;2:1477-86.

29. Thomas JS, Julian HS, Green RV, et al. Histopathology of breast carcinoma following neoadjuvant systemic therapy: a common association between letrozole therapy and central scarring. Histopathology 2007;51:219-26. 